\title{
Design Solutions for Compact High Current Pulse Transformers for Particle Accelerators' Magnets Powering
}

\author{
Davide Aguglia, Jean-Marc Cravero \\ CERN, Geneva, Switzerland, \\ Renato Rebeschini, Salvatore Iovenio ; Carlo Russo \\ TRASFOR S.A., Molinazzo di Monteggio, Switzerland
}

Keywords: «Transformer», «Pulsed power converter», «Particle accelerator».

\begin{abstract}
High current pulse transformers are widely used in particle accelerators for feeding pulsed magnets. Usually they need to be installed in very confined spaces, thus requiring compact design solutions. Several magnetic bias solutions are explored with the objective of reducing the core volume and total cost of the converter-transformer system. A concrete example is illustrated via the optimal design of two variants of the same pulse transformer, one with an air-gap and the other without air-gap but with an active magnetic reset. Results show that several solutions exist in order to optimize the volume of pulse transformers; however, this is always done at the expense of a more complex power converter topology or increased magnet volume.
\end{abstract}

Presented at: EPE 2015, 7-10 September 2015, Geneva, Switzerland

Geneva, Switzerland

October, 2015 


\title{
Design Solutions for Compact High Current Pulse Transformers for Particle Accelerators' Magnets Powering
}

\author{
Davide Aguglia ${ }^{1}$, Jean-Marc Cravero ${ }^{1}$, Renato Rebeschini ${ }^{2}$, Salvatore Iovieno ${ }^{2}$, \\ and Carlo Russo ${ }^{2}$ \\ ${ }^{1}$ CERN-EUROPEAN ORGANIZATION FOR NUCLEAR RESEARCH \\ 1217 Geneva 23, Switzerland \\ Tel.: $+41 /(0)-22.767 .55 .49$. \\ E-Mail: Davide.Aguglia@cern.ch \\ URL: http://www.cern.ch \\ 2 TRASFOR S.A. \\ 6995 Molinazzo di Monteggio, Switzerland \\ Tel.: $+41 /(0)-58.588 .44 .00$. \\ E-Mail: Renato.Rebeschini@ch.abb.com \\ URL: http://www.trasfor.ch
}

\section{Keywords}

«Transformer», «Pulsed power converter», «Particle accelerator».

\begin{abstract}
High current pulse transformers are widely used in particle accelerators for feeding pulsed magnets. Usually they need to be installed in very confined spaces, thus requiring compact design solutions. Several magnetic bias solutions are explored with the objective of reducing the core volume and total cost of the converter-transformer system. A concrete example is illustrated via the optimal design of two variants of the same pulse transformer, one with an air-gap and the other without air-gap but with an active magnetic reset. Results show that several solutions exist in order to optimize the volume of pulse transformers; however, this is always done at the expense of a more complex power converter topology or increased magnet volume.
\end{abstract}

\section{Introduction}

In particle accelerators, pulse transformers are used to supply several kinds of electro-magnets. The pulsed operation mode is often required for magnets utilised for beam injection and ejection of circular accelerators [1] (synchrotrons). In many accelerators, such as Linacs [2], and small to medium energy synchrotrons, the magnetic field is only periodically required for short periods of time. To reduce the global power consumption of such accelerators the growing tendency is to adopt a pulsed powering scheme [3] for such magnets.

The current and voltages which have to be applied to this kind of electro-magnets often require an adaptation transformer in order to utilise standard power converter's ratings, imposed by the selected semiconductor device technology (IGBT, thyristors, MOSFET, IGCT, etc.) and converter topology. As the semiconductor current capability decreases rapidly as their voltage rating increases, the pulse transformers are often used to deliver high current at their secondary [4]. Furthermore, the magnetic force used to deflect the particle beam is proportional to the magnet current and high pulsed currents allow large deflection angles that are required for the injection or ejection of particle beams. The general powering layout and typical magnet waveforms are illustrated in Fig. 1. 


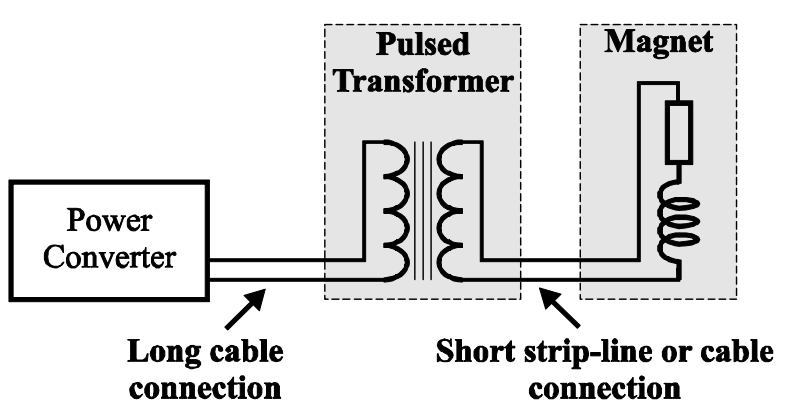

b)

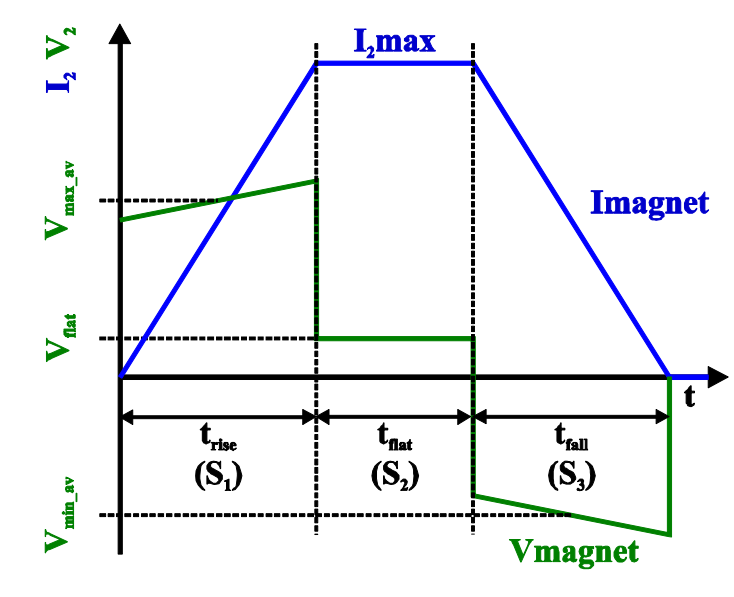

Fig. 1: a) - General magnet powering layout, and b) - Typical magnet current and voltage waveforms.

Usually, the accelerator magnets are located in a dedicated tunnel and the associated power converters are not installed in their immediate vicinity because of radiations issues, converter maintenance aspects, etc... However, to optimise the magnet powering system, the distance between magnet and transformer should be minimised [5] and, ideally, the pulse transformer should be located as close as possible to the magnet. This requirement often leads to integration issues since the space inside the accelerator tunnel is usually optimised and limited. Such difficulties are particularly critical during accelerators renovation/upgrades phases, where higher magnetic energies and electrical power are required, while keeping the same civil engineering infrastructures. In these situations, as well as in new accelerators designs, it is of primary importance to find compact and efficient solutions to minimize the volume of installed material in the accelerator gallery.

Considering these integration issues, this paper proposes and analyses possible solutions aiming at minimizing high current pulse transformer's volume. In particular, solutions for minimizing the magnetic core volume are analysed.

\section{DC bias and active reset solutions for core volume minimisation}

The core magnetic material utilisation can be illustrated by analysing its magnetic (induction $B$ vs. Magnetic field $H$, or $B-H$ ) dynamic characteristic during the pulse transformer nominal operation. For illustration purposes an arbitrary $B-H$ dynamic characteristic, available in MatLab ${ }^{\circledR}$ Simulink, has been utilised.

Figure 2 a) presents the simplest case where the pulse transformer is operated with a repetitive sequence of current and voltage pulses as illustrated in Fig. 1 b). Supposing a starting point in the $B-H$ characteristic at $\mathrm{B}=0 \mathrm{~T}$ and $\mathrm{H}=0 \mathrm{~A} / \mathrm{m}$, the red curve represents the very first pulse, forming cycle 1 (in red) on the $B-H$ characteristic. One can notice that this cycle is divided in 2 segments; the first one with increasing $\mathrm{B}$ and $\mathrm{H}$, corresponding to sectors $\mathrm{S} 1$ and $\mathrm{S} 2$ of Fig. $1 \mathrm{~b}$ ), and the second one with decreasing $\mathrm{B}$ and $\mathrm{H}$, corresponding to sequence $\mathrm{S} 3$ of Fig. $1 \mathrm{~b}$ ). Within the increasing segment the magnetizing current increases (x-axis of graphics in Fig. 4), whereas within the decreasing segment, the magnetizing current decreases to zero. In this case the magnetizing current is never negative; therefore the half-left plane of the $B-H$ characteristic is never exploited. It can be noted that, after the first pulse, the magnetic cycle ends with a given remanent magnetization. Therefore, the next pulse will impose the next magnetic cycle to start from a different point where $\mathrm{B} \neq 0 \mathrm{~T}$. Furthermore, during these first cycles, the positive $\Delta \mathrm{B}$ is greater than the negative one. After this magnetic flux building-up sequence, given by the hysteresis effect, stable operation is reached, where positive and negative magnetic excursions are equivalent. However, this stabilisation will always take place near the saturation region, leading to an increased magnetizing current of the pulse transformer. One can notice how the nominal and stable operation of the pulse transformer is achieved using a very small fraction of magnetic excursion compared to the $B-H$ characteristic potential excursion. 
One way to overcome this issue, and better utilise the potential magnetic flux excursion, consists in modifying the $B-H$ characteristic such that the remanent flux is decreased. This goal can be achieved by selecting a different magnetic material and/or by inserting an air-gap in the magnetic core. The latter solution is commonly used in several applications. Figure $4 \mathrm{~b}$ ) illustrates this concept, where the new $B-H$ characteristic includes an air-gap. After operation stabilisation (3 pulses/magnetic cycles), it can be noted how the magnetic flux swing (and induction B swing) is increased. With a proper air-gap dimensions selection, it is possible to utilize almost the full half positive magnetic flux swing, with no drastic increase in the magnetizing current.

In order to improve the utilization of the transformer core material and to further reduce the size and cost of the pulse transformers, two possible solutions could be adopted: the first one is the use of a DC bias circuit (Fig. $2 \mathrm{c}$ )) and the second is the use of a bipolar pulsed converter to actively reset the magnetic core of the transformer (Fig. $2 \mathrm{~d}$ )).

a)

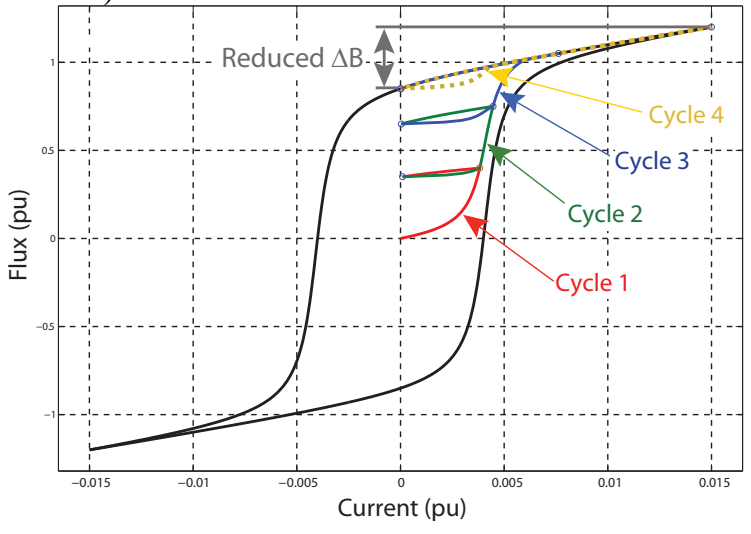

c)

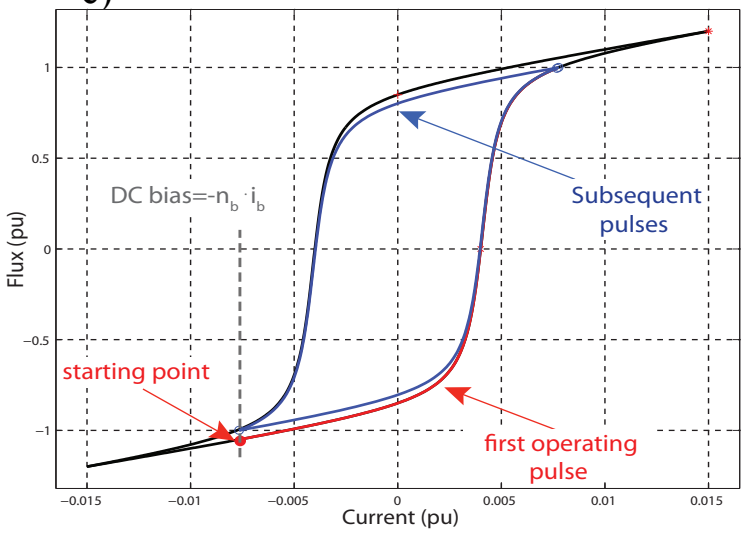

b)

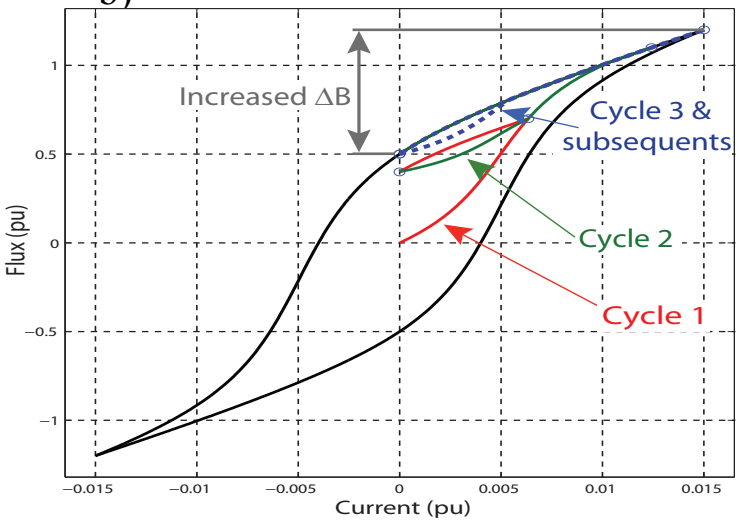

d)

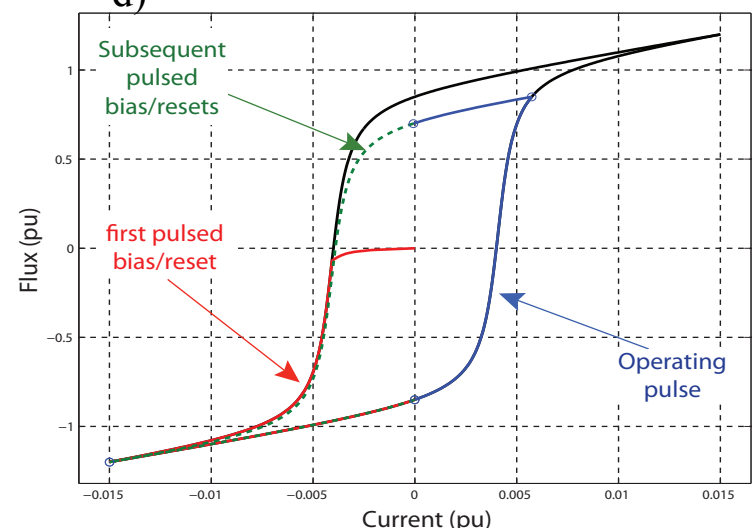

Fig. 2: a) - $B-H$ dynamical characteristic utilization with no bias solution; b) - with no bias and with an air-gap; c) - with DC bias; d) - with pulsed bias / active reset.

A solution consists in foreseeing a third winding, called bias winding, in which a DC current is imposed in order to reset the flux in the core to a value close to the maximum negative saturation. In this case, the available flux excursion will be significantly improved when compared to a unipolar use of the core (Fig. 2 a) \& b)). Because of its simplicity, this option is widely used in many domains like pulse transformers for klystron modulators [6]. Nevertheless, this solution requires the implementation of an additional circuit composed of a DC power supply and a large inductance to stabilise the DC bias current during the main operating pulse.

A different option consists in the use of a pulsed current in the opposite direction of the operating pulsed current to drive the core flux in its negative region, close to saturation, as illustrated in Fig. 2 b). Compared to the previous solution using a DC bias winding, the magnetic flux swing is slightly 
reduced since the operating cycle starts from the negative maximum remanence instead from deep negative saturation. This option has been well documented for switch-mode converters using the forward topology [7, 8]. In the field of high power pulsed systems, this solution has also been investigated for the design of the pulse transformers for klystron modulators [9]. Considering the design of pulsed transformers for particle beam deflection, a possible solution consists in the use of a bipolar power converter to provide an optimized use of the core material. Although this solution is not always possible, it could be implemented for powering magnets installed in transfer lines, injection and ejection regions, where the beam is present during a small lapse of time. Consequently, the active reset of the transformer is possible without any perturbations as regards to the normal beam operation.

Another solution would consist in integrating a permanent magnet in the transformer core, biasing the magnetic flux. This solution has been investigated for small pulse transformers [10], and could also be an interesting, but costly, alternative in high current pulse transformers.

\section{Experimental validation}

Experimental tests have been performed in order to evaluate and quantify the possible improvements when such an optimization of the use of the magnetic core is implemented. The experimental setup is based on the use of a fast pulsed current source that supply an inductive load using a step-up (in current) pulse transformer. The power source delivers quasi-trapezoidal current pulses with high precision flat-top. The experimental setup parameters are summarized in Table I, and pictures of the experimental test-bed and pulse transformer are illustrated in Fig. 3 a) \& b).

a)

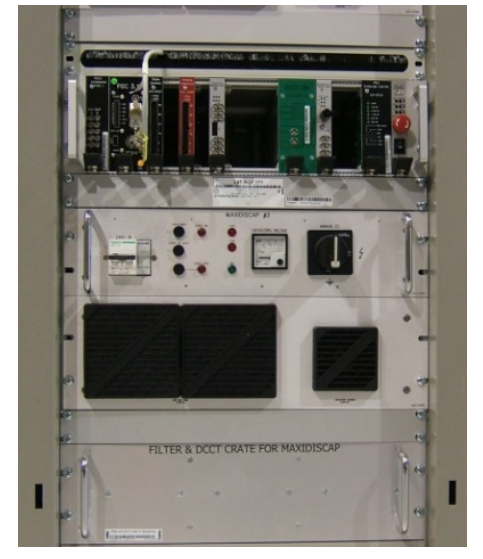

b)

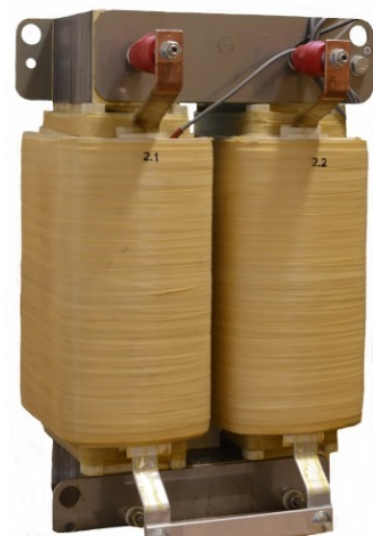

Fig. 3: a) - View of the power converter used for experimental validation; b) - Picture of the pulse transformer used for experimental validation.

Table I: Experimental setup parameters

\begin{tabular}{|l|l|r|l|}
\hline & Parameters & Value & Unit \\
\hline \multirow{2}{*}{ Converter ratings } & Maximum voltage & 1000 & $\mathrm{~V}$ \\
& Maximum current & 300 & $\mathrm{~A}$ \\
\hline \multirow{3}{*}{$\begin{array}{l}\text { Pulse transformer } \\
\text { ratings }\end{array}$} & Primary turns number & 120 & - \\
& Secondary turns number & 24 & - \\
& Maximum secondary current & 1250 & $\mathrm{~A}$ \\
\hline \multirow{2}{*}{ Load parameters } & $\mathrm{L}$ & 210 & $\mu \mathrm{H}$ \\
& $\mathrm{R}$ & 0.15 & $\Omega$ \\
\hline
\end{tabular}


At first, the pulse transformer is used only in its positive flux region: the secondary current is set to $520 \mathrm{~A}$ during the flat-top and the repetition period is set to 3.6s to allow the demagnetization of the transformer. As shown in Fig. 4 a), the peak magnetizing current (reflected to the primary side of the transformer) reaches $38 \mathrm{~A}$ and the pulse transformer is used in its saturation region. This operation corresponds to the use of the magnetic material that is illustrated in Fig. 2 a).

In order to optimize the transformer core utilization, the power converter then delivers alternative current pulses with $+520 \mathrm{~A}$ and $-520 \mathrm{~A}$ during the flat-tops. In this case, as shown in Fig. $4 \mathrm{~b}$ ), the magnetizing current is significantly reduced and the transformer is not operated in its saturation region anymore. Consequently, its magnetic core volume can be minimized with the same operational performances.
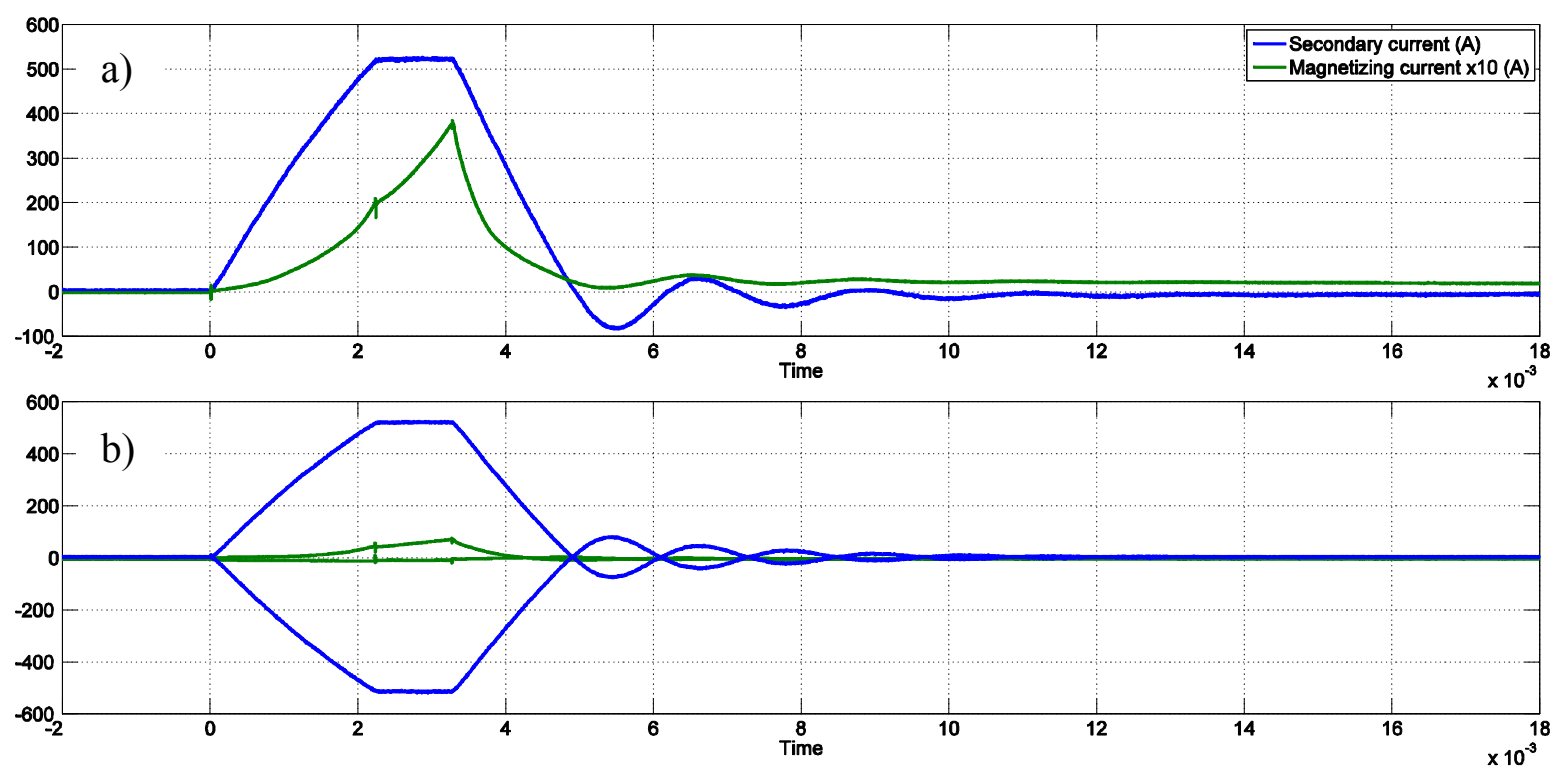

Fig. 4: a) - Secondary current (blue) and magnetizing current (green) of the pulse transformer with a unipolar use of the magnetic core; b) - secondary current (blue) and magnetizing current (green) of the pulse transformer when a negative current pulse is used to reset the magnetic core.

As a second experimental test, the core is first saturated in the negative region with the application of a sequence of repetitive negative current pulses, in order to fully reset the magnetic core and allow the maximum excursion from negative to positive flux. Then, a series of positive current pulses is applied. In this case, the whole hysteresis curve is covered from the negative saturation region to the positive one, and several magnetic cycles are necessary to reach saturation (as in Fig. 2 a)), however starting from deep negative saturation. The corresponding waveforms are given in Fig. 5, showing that a high negative current pulse can reset the core for several following positive pulses.

This feature could be interesting when multiple injection/ejections of particle beams are used with no time to produce negative pulses between operational ones. Therefore the reset of the transformer core can be performed well in advance and several current pulses can be then provided without any saturation phenomenon. However this solution would imply to increase the RMS current in the transformer's windings as well as in the magnet.

\section{Application to a specific pulsed transformer design}

The analyses presented in this paper are applied to concrete projects where the need of minimizing the pulse transformer size is essential. A specific set of pulse transformers, specified in Table II, has to be designed for the upgrade of CERN's Proton Synchrotron Booster (PSB) [11]. 

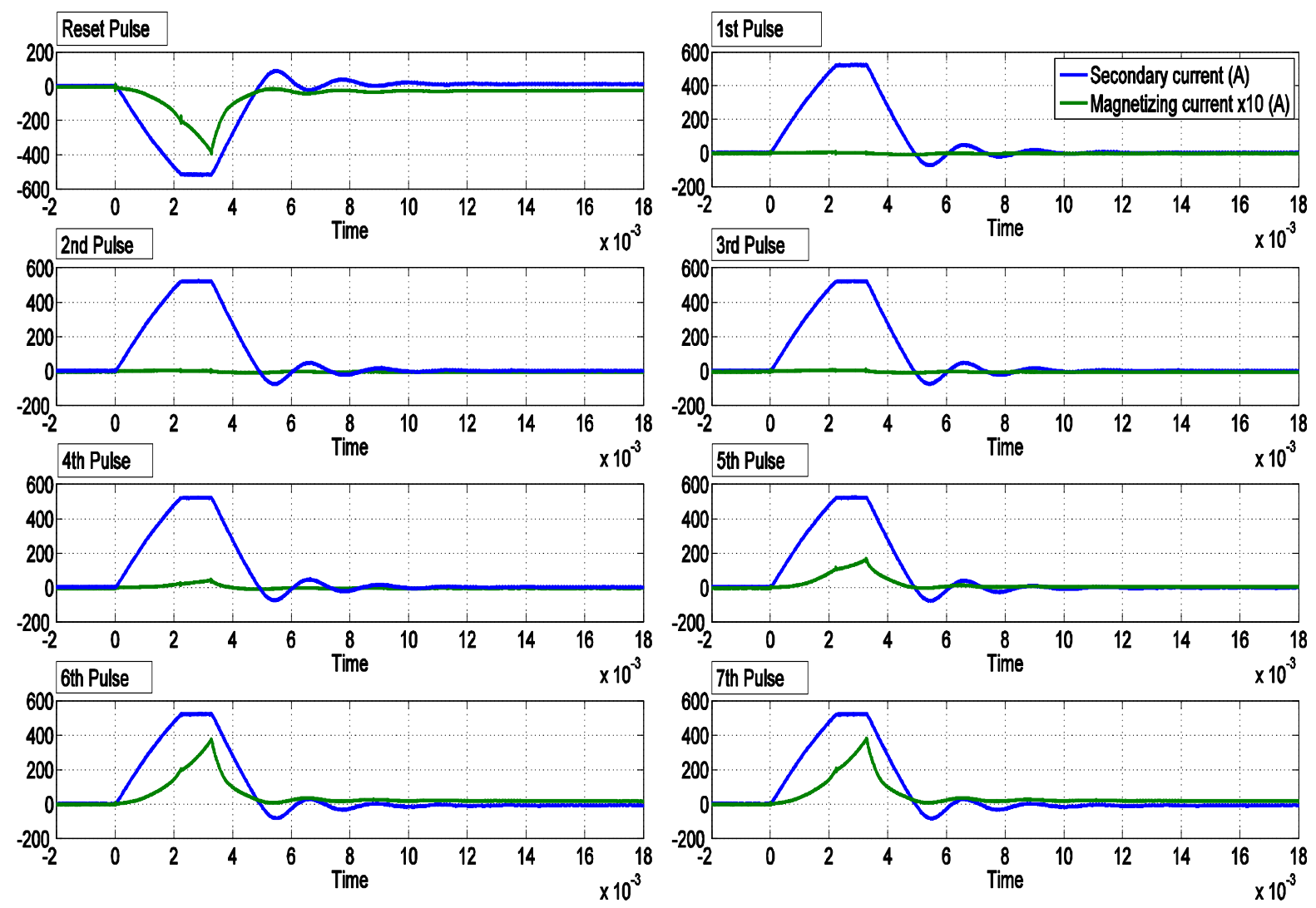

Fig. 5: Evolution of the secondary current (blue) and 10x magnetizing current (green) of the pulse transformer when repetitive negative current pulses are used to drive the magnetic core in its negative saturation region and a sequence of 8 positive current pulses are then applied.

\section{Table II: Pulse transformer specification for design examples}

\begin{tabular}{|l|c|r|l|}
\hline Parameter & Symbol & Value & Unit \\
\hline Maximal secondary current & $\mathrm{I}_{2 \max }$ & 3.4 & $\mathrm{kA}$ \\
\hline $\mathrm{I}_{2} / \mathrm{I}_{1}$ transformer ratio & $\mathrm{k}$ & 4 & - \\
\hline Minimal magnetizing inductance & $\mathrm{L}_{\mathrm{m}}$ & 100 & $\mathrm{mH}$ \\
\hline Current rise-time & $\mathrm{t}_{\text {rise }}$ & 5 & $\mathrm{~ms}$ \\
\hline Current flat-top duration & $\mathrm{t}_{\text {flat }}$ & 2 & $\mathrm{~ms}$ \\
\hline Current fall-time & $\mathrm{t}_{\text {fall }}$ & 5 & $\mathrm{~ms}$ \\
\hline Average voltage during rise-time & $\mathrm{V}_{\text {max_av }}$ & 100 & $\mathrm{~V}$ \\
\hline Average voltage during flat-top & $\mathrm{V}_{\text {flat }}$ & 75 & $\mathrm{~V}$ \\
\hline Average voltage during fall-time & $\mathrm{V}_{\text {min_av }}$ & -45 & $\mathrm{~V}$ \\
\hline
\end{tabular}

Two optimal design procedures are carried out for comparing the following pulse transformer solutions:

1. With magnetic core air-gap and no bias action,

2. Without magnetic core air-gap and pulsed bias/reset action.

With the objective of minimizing the volume, the leakage inductance, and respecting the minimal magnetizing inductance (Table II), the two variants of this transformer have been optimized and 
design results are presented in table III. Figure 6 illustrates the pulse transformer of the solution with air-gap and defines the overall dimensions.

Table III: Pulse transformers design results with and without core air-gap

\begin{tabular}{|l|c|c|c|l|}
\hline Parameter & Symbol & With air-gap & Without air-gap & Unit \\
\hline Maximal magnetic induction & $\mathrm{B}_{\max }$ & +1.4 & +1.3 & $\mathrm{~T}$ \\
\hline Minimal magnetic induction & $\mathrm{B}_{\min }$ & +0.2 & -0.7 & $\mathrm{~T}$ \\
\hline Magnetic induction excursion & $\Delta \mathrm{B}$ & 1.2 & 2 & $\mathrm{~T}$ \\
\hline Magnetic core weight & $\mathrm{M}_{\mathrm{fe}}$ & 374 & 223 & $\mathrm{~kg}$ \\
\hline Copper winding weight & $\mathrm{M}_{\mathrm{cu}}$ & 76 & 47 & $\mathrm{~kg}$ \\
\hline Total weight & $\mathrm{M}_{\mathrm{tot}}$ & 560 & 340 & $\mathrm{~kg}$ \\
\hline Overall length & $\mathrm{L}$ & 590 & 500 & $\mathrm{~mm}$ \\
\hline Overall height & $\mathrm{H}$ & 540 & 470 & $\mathrm{~mm}$ \\
\hline Overall depth & $\mathrm{D}$ & 515 & 475 & $\mathrm{~mm}$ \\
\hline Volume & $\mathrm{L} \times \mathrm{H} \times \mathrm{D}$ & 0.164 & 0.112 & $\mathrm{~m}^{3}$ \\
\hline
\end{tabular}
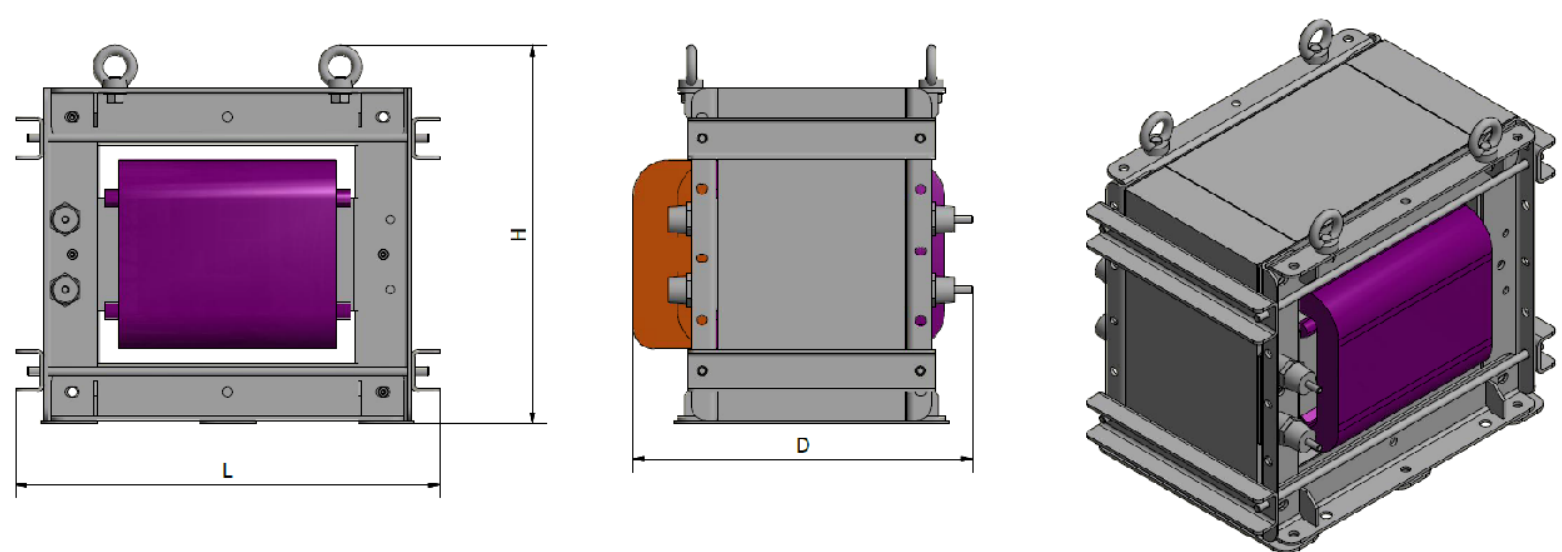

Fig. 6: 3D illustration of the optimized pulse transformer solution with air-gap, and overall dimensions definition (L, H, and D).

The two solutions use the same core magnetic material. Notice that the air-gapped solution has a maximal magnetic induction excursion of $1.2 \mathrm{~T}$ compared with the $2 \mathrm{~T}$ of the solution with no air-gap. The overall weight and dimensions are significantly reduced.

One has to mention that in the solution without air-gap, and with a pulsed bias action, the overall RMS current circulating in the transformer and in the magnet windings is increased (due to a supplementary bias/reset pulse within the same cycle). This implies an increase in copper total cross-section in the pulse transformer as well as in the magnet. The total copper volume increase in the pulse transformer is compensated by the shorter average turn length (due to magnetic core size reduction); however, the magnet winding cross sectional area shall be increased according to the RMS current increase. This leads to a compromise between the pulse transformer and magnet volumes. To overcome this drawback one can imagine two solutions:

1. Use of a DC bias winding: in this case there is no need for a reset pulse; however, the pulse transformer volume will be slightly increased by the addition of a dedicated bias winding.

2. Insertion of a diode in the secondary circuit: this solution allows keeping a pulsed reset operation and forcing the de-magnetization/reset current to flow in the pulse transformers 
primary winging only. The drawback of this solution is that the high current/low voltage diode is installed in a harsh environment (e.g. heat, radiation, etc.), where maintenance and repairs are made difficult.

\section{Conclusion}

Compared to existing solutions, high current pulse transformer design can be further optimized such that their volume and cost can be minimised. Such an optimisation can be particularly appreciated in applications where space for placing these devices is limited. In pulsed applications, the pulse transformer volume is predominantly driven by the magnetic core material rather than the windings. It is clear that reducing the core volume by optimising the associated $B-H$ characteristic utilization leads to a direct overall pulse transformer volume reduction.

Several solutions are explored and magnetic DC bias or active reset methods can appreciably help in reducing volume and cost. The DC bias solution presents the advantage of maximising the utilisation of the magnetic material. However, the transformer design shall integrate an additional winding which has to be supplied by an additional DC power converter. Furthermore, special care must be taken in stabilizing the DC bias current during the operational current pulse. Indeed, during the magnetic flux swing, voltage is induced in the bias winding and care must be taken to protect the required low voltage DC bias power converter. The active reset, or inverse pulse reset, method has the advantage of keeping the pulse transformer design simple. In this case, the bias winding is the primary winding itself. However, compared to the DC bias solution, this method allows pre-magnetizing the magnetic circuit to the negative remanent flux only. Furthermore, special care must be given to the RMS current increase in the transformer and in the magnets due to the additional inverse/reset pulse during the cycle.

Finally, each power converter-pulse transformer-magnet circuit should be globally analyzed to decide whether an air-gapped solution, or a bias (DC or pulsed) solution should be adopted. Compromises will always occur between the power converter complexity and cost and the pulse transformer and magnet volumes and costs.

\section{References}

[1] Bordry F.: Power converters for particle accelerators, European Conference on Power Electronics and Applications (EPE), 2005, pp. 30

[2] Wilson E. J. N.: An Introduction to Particle Accelerators, Oxford University Press, 2001, p. 252

[3] Voelker F.: Pulsed capacitor discharge power converter: an introductory overview, CERN Accelerator School: Power Converters for Particle Accelerators, Montreux, Switzerland, CERN, March 1990

[4] Cravero J. M., Maire G., Royer J.P.: High current capacitor discharge power converters for the magnetic lenses of a neutrino beam facility, European Conference on Power Electronics and Applications (EPE), 2007, pp. $1-9$

[5] Aguglia D.: Pulse transformer design for magnet powering in particle accelerators, European Conference on Power Electronics and Applications (EPE), 2013, pp. 1-9

[6] Bortis D., Biela J., Kolar J. W.: Optimal Design of a DC Reset Circuit for Pulse Transformers, IEEE Applied Power Electronics Conference (APEC), 2007, pp. 1171-1177

[7] Vinciarelli P.: Optimal resetting of the transformer's core in single ended forward converters, U.S. Patent N4,441,146, Apr.1984

[8] Li Q., Lee F.C., Jovanovic M. M.: Design considerations of transformer DC bias of forward converter with active-clamp reset, IEEE Applied Power Electronics Conference (APEC), 1999, pp. 553-559

[9] Bortis D., Biela J., Kolar J. W.: Design and Control of an Active Reset Circuit for Pulse Transformers, IEEE International Power Modulators and High Voltage Conference, 2008, pp. 392-395

[10] Mizumo T., Takata Y., Matsumoto M.: A Hybrid Pulse Transformer with Permanent Magnets, IEEE Transactions on Magnetics, Vol. 41 no 5, 2005, pp. 3982-3984

[11] LHC Injectors Upgrade, Technical Design Report, Vol. 1: Protons, CERN-ACC-2014-0337, CERN, Geneva, 2014, $613 \mathrm{p}$. 